\title{
Ramsey Interference with Single Photons
}

\author{
Stéphane Clemmen, ${ }^{1, *}$ Alessandro Farsi, ${ }^{1}$ Sven Ramelow, ${ }^{2}$ and Alexander L. Gaeta ${ }^{1,3}$ \\ ${ }^{1}$ School of Applied and Engineering Physics, Cornell University, Ithaca, New York 14853, USA \\ ${ }^{2}$ Faculty of Physics, Humboldt-University Berlin, Berlin 12489, Germany \\ ${ }^{3}$ Department of Applied Physics and Applied Mathematics, Columbia University, New York, New York 10027, USA
}

(Received 13 June 2016; published 23 November 2016)

\begin{abstract}
Interferometry using discrete energy levels of nuclear, atomic, or molecular systems is the foundation for a wide range of physical phenomena and enables powerful techniques such as nuclear magnetic resonance, electron spin resonance, Ramsey-based spectroscopy, and laser or maser technology. It also plays a unique role in quantum information processing as qubits may be implemented as energy superposition states of simple quantum systems. Here, we demonstrate quantum interference involving energy states of single quanta of light. In full analogy to the energy levels of atoms or nuclear spins, we implement a Ramsey interferometer with single photons. We experimentally generate energy superposition states of a single photon and manipulate them with unitary transformations to realize arbitrary projective measurements. Our approach opens the path for frequency-encoded photonic qubits in quantum information processing and quantum communication.
\end{abstract}

DOI: 10.1103/PhysRevLett.117.223601

The two-level model represents the most fundamental quantum system and is used to describe a wide variety of physical systems. Ramsey interferometry, magnetic resonance imaging, and electron-spin resonance spectroscopy are governed by two-level system dynamics involving, respectively, molecular-atomic levels, nuclear spin, and electronic spin. In those examples, the coupling between energy levels is achieved using electromagnetic fields that can be tailored at will and allows for many advanced techniques such as adiabatic elimination and stimulated Raman adiabatic passage in a higher dimensional atomic system, or spin locking in NMR. Quantum interference involving systems in a superposition of different energies is at the heart of fundamental and applied physics and has been highly useful in increasing the accuracy of time measurement from the first idea of using NMR suggested by Rabi in 1945 [1,2] to the first atomic clock relying on Ramsey interferometry [3-5], recently renewed by using trapped single ions [6]. Ramsey interferometry on single Rydberg atoms has allowed the nondestructive measurement of the number of photons in a cavity [7], single spin manipulation using the same techniques constitutes one of the most promising routes towards quantum processing [8-10], and Ramsey interferometry also enables building two-qubit quantum gates [11]. Matter-wave interferometers using collective energy levels of atoms in a Bose-Einstein condensate have also been demonstrated [12] and used to measure gravity down to record breaking precision [13]. Nevertheless, a fundamental quantum system that has not been extensively studied in the context of discrete two-level energy systems is a single photon. Translating those studies to a photonics system can be implemented by controlling light with light using nonlinear optical phenomena. For classical light the analogy between atomic or molecular optics and nonlinear optics is well known [14] and there are various cases where the complex dynamics of light propagation in a nonlinear medium can be simplified to the coherent evolution of a two-level system. For a quantum of light a bichromatic qubit is a photon whose frequency can be one of two possible colors. A key requirement is to manipulate the frequency states of single photons while preserving their coherence. This coherence allows for transitions between the two frequencies that mimic Rabi oscillations $[15,16]$.

In this Letter, we demonstrate the manipulation of single photons and their corresponding position on the energy Bloch sphere. We show the resulting quantum interference associated with this two-level quantum system. On the Bloch sphere, polar rotations are achieved using a phase sensitive wave-mixing process known as Bragg scattering (BS) fourwave mixing (FWM) that translates the frequency of the initial state to a new frequency without adding noise. Azimuthal rotations are implemented by imparting tunable physical delays on the single photon. This approach to encoding quantum information onto the energy degree of freedom of single photons represents an important advance on previously introduced frequency-encoded quantum information reported by Merolla [17-20] or Zeilinger [21] and temporal modes introduced by Brecht [22-26] since in our case such a qubit can be implemented as a coherent superposition of two colors. Such a representation was not straightforward to realize in earlier works [17-21]. It has been shown that photons from different frequencies interfere at the single quantum level [17] and that such a two-party system can violate Bell inequalities [18]. However, those studies do not allow quantum logic operations in a two-dimensional Hilbert space unless a more complex definition of a frequency-encoded qubit is introduced [19]. A closer approach [22-26] has been realized using temporal modes encoded on a single broader frequency span. Interestingly, the authors aiming at sorting temporal modes also introduce a toolbox made of linear quantum gates for their temporal modes. 
The core of our work is the efficient and low-noise frequency conversion of single photons that was proposed two decades ago [15,27] and for which various successful studies using $\chi^{(2)}$ and $\chi^{(3)}$ nonlinear interactions have been demonstrated [28-37]. Figure 1 depicts the minimum set of operations in a Ramsey interferometer as rotations on a Bloch sphere where the poles represent states of the two different energy levels. In a Ramsey interferometer, the two-level system undergoes subsequent identical interactions with resonant pulses of area $\pi / 2$ that are separated by a noninteracting interval of free evolution. For the photonic realization we first define two discrete frequencies and prepare the photon in one of them, $\left|\nu_{s}\right\rangle$, that we associate with the south pole of the Bloch sphere. A $\pi / 2$ pulse transforms this state into a superposition of the form $2^{-1 / 2}\left(\left|\nu_{s}\right\rangle+\left|\nu_{i}\right\rangle\right)$ corresponding to a $\pi / 2$ polar rotation in the $O_{x, z}$ plane along the meridian of the Bloch sphere. The bichromatic qubit is subsequently left free to evolve for a time $T$ resulting in an azimuthal rotation. This corresponds to the system acquiring a relative phase $\pi=2 \pi T \delta \nu$ due to the precession between the two levels of different frequency $\delta \nu$. Finally, a second $\pi / 2$ pulse is applied that transforms the superposition state into the final state depending critically on the imparted phase $\phi$. The projective measurement consists of detecting whether the photon has a frequency $\nu_{s}$ or $\nu_{i}$ and therefore reveals information on the phase $\phi$. In order to prepare any state of a bichromatic qubit, that is, implement the scheme depicted in Fig. 1, our toolbox requires four elements: (A) a bichromatic qubit defined by two-dimensional Hilbert space $\left\{\left|\nu_{s}\right\rangle,\left|\nu_{i}\right\rangle\right\}$, (B) a photon frequency converter capable of transferring the eigenvector back and forth $(\pi / 2$ pulse), (C) control on the relative phase $\phi$, and (D) a measurement of the energy of the final state. To define a bichromatic qubit (A) and initialize it on a pole of the Bloch sphere, we isolate a single photon at a given frequency (A) $\nu_{s}$ by using a frequency heralded photon source. Photon pairs are generated via spontaneous down conversion over frequencies $\nu_{s}$ and $\nu_{\text {heralding }}$ so that energy is conserved, $\nu_{s}+\nu_{\text {heralding }}=\nu_{\text {pump }}$, where $\nu_{\text {pump }}$ is the fixed frequency of a pump beam. By spectrally filtering the broad flux of the heralding photons, thus imposing a given value to $\nu_{\text {heralding }}$

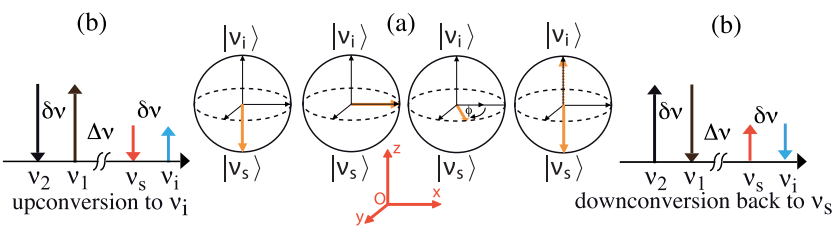

FIG. 1. (a) Principle of a Ramsey interference based on discrete energy levels of photons depicted as rotations on the Bloch sphere. (b) Up- or down-conversion for a particular set of frequencies $\left\{\nu_{1}, \nu_{2}, \nu_{i}, \nu_{s}\right\}$ via Bragg scattering four-wave mixing corresponds to polar rotation on the Bloch sphere. $\delta \nu=\nu_{1}-$ $\nu_{2}=\nu_{i}-\nu_{s}$ and $\Delta \nu=\nu_{s}-\nu_{1}$. prior to its detection in a single-photon detector, the partner photon at frequency $\nu_{s}$ is characterized in time and frequency. Indeed, as the heralding and heralded photons are created as a single event, the timing of a heralded photon is known down to the timing resolution of the heralding detector while its spectrum is bounded by energy conservation and the bandwidth of the bandpass filter placed in front of the heralding detector.

To manipulate the bichromatic qubit we perform quantum frequency conversion on the generated single photons. Quantum frequency conversion has been demonstrated using second order $\chi^{(2)}$ nonlinearities either via sumfrequency generation [15,27,32-36], difference-frequency generation [16,38,39], or electro-optic modulation [17-20]. In contrast, Bragg scattering four-wave mixing is a third order $\chi^{(3)}$ nonlinear process that has also been shown to be an effective [28-31] way of achieving quantum frequency conversion applicable to many types of waveguide platforms. A major advantage of BS FWM over sum-frequency generation and electro-optic modulation is an easier to satisfy constraint concerning the frequencies involved in the conversion. Indeed, for BS FWM the principal constraint lies in the phase matching condition while sum frequency generation has the additional requirement that interacting fields have to be at least one octave apart. Electro-optic modulation is bounded to the other extreme to a frequency shift in the gigahertz range because of the electric modulation involved. In our experiment we employ a BS-FWM configuration that is depicted in Fig. 1(b) in which two strong fields (referred to as pump beams) $E_{1}\left(\nu_{1}\right)$ and $E_{2}\left(\nu_{2}\right)$ are frequency detuned by $\delta \nu$. This FWM process annihilates a pump photon from the field $E_{2}\left(\nu_{2}\right)$ and the target photon at the signal frequency $\nu_{s}$ for creating one photon on the field $E_{1}\left(\nu_{1}\right)$ and one at the idler frequency $\nu_{i}=\nu_{s}+\delta \nu$ such that the total energy is conserved. A critical aspect of BS FWM as compared to other FWM processes is that it provides a coherent or phase sensitive coupling between the two frequencies without adding noise and thus creates a proper bichromatic photon qubit. In the ideal case of a perfectly phase-matched process, the coherent coupling induced by the FWM BS is expressed by the following coupled equations for the annihilation operators $\hat{a}_{s}(z)$ and $\hat{a}_{i}(z)$ for the signal and idler fields, respectively:

$$
\left[\begin{array}{l}
\hat{a}_{s} \\
\hat{a}_{i}
\end{array}\right]=\left[\begin{array}{cc}
\cos 2 \gamma P z & i e^{i \theta} \sin 2 \gamma P z \\
i e^{-i \theta} \sin 2 \gamma P z & \cos 2 \gamma P z
\end{array}\right]\left[\begin{array}{l}
\hat{a}_{s}(0) \\
\hat{a}_{i}(0)
\end{array}\right],
$$

where $\gamma$ is the nonlinear coefficient, $z$ is the propagation distance, and $P=\left|E_{1}\right|^{2}=\left|E_{2}\right|^{2}$ is the power of the two pump beams with a relative phase $\theta$. From Eq. (1), it is seen that BS FWM produces a rotation in the frequency Hilbert space $\left\{\left|\nu_{s}\right\rangle,\left|\nu_{i}\right\rangle\right\}$ such that $\gamma P z=\pi / 8$ corresponds to a $\pi / 2$ rotation. In addition, Eq. (1) exhibits a phase dependence $\theta$ originating from the pump fields. This implies extra care to 
preserve those phase relationships between subsequent BSFWM processes. Departures from the ideal case of a noiseless unity-efficiency process, such as the effects of imperfect phase matching, higher-order BS, and spurious sources of noise, are discussed in the Supplemental Material [40]. A controllable relative phase between two optical frequencies $(\mathrm{C})$ can be readily produced by propagating the bichromatic photon qubit over a length-tunable delay line. Measurement of the final frequency of the bichromatic photon (D) simply requires separating the two spectral components into two paths using a dispersive element and detecting in which path the photon is present using single photon detectors.

The implementation of those four primary components (A)-(D) is illustrated as insets in Fig. 2. Figure 2(a) shows the state preparation in which a single photon is heralded by detection with a silicon avalanche photodiode of its partner photon generated at $\lambda_{\text {heralding }}=940 \mathrm{~nm}$. The heralding photon is spectrally filtered so that its partner heralded photon is spectrally defined at $\lambda_{s}=1283 \mathrm{~nm}$ with a bandwidth of $0.5 \mathrm{~nm}$. To align, synchronize, and characterize the setup, we use a tunable laser with a variable attenuator to produce a weak coherent field with less than 0.1 photon per gate. The signal wavelength and bandwidth are selected to accommodate the frequency converter [Fig. 2(b)]. The second order correlation $g^{(2)}(t=0)$ of our single photon source is limited to 0.23 due to a tradeoff involving a reasonable dark count level and a reasonable low probability of multipair emission. The design of the frequency converter is depicted in Fig. 2(b) (see Secs. S1-S6 of the Supplemental Material [40] for an in depth design review of the frequency converter). It is exclusively built of optical fibers. The pump beams are generated from nanosecond-pulsed laser diodes and amplified via erbium doped fibers, then overlapped (spatially, temporally, and in polarization) with the single photons via fiber wavelength multiplexers into a nonlinear dispersion shifted fiber where the quantum frequency conversion takes place (see Fig. S3 of the Supplemental Material [40] for its group velocity dispersion). To minimize Raman noise, the fiber is cooled down to cryogenic temperature (see Fig. S1 of

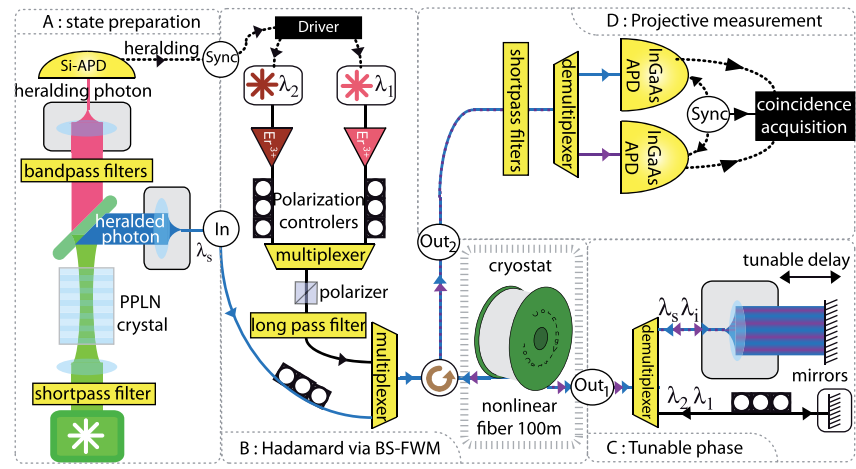

FIG. 2. (a)-(d) Experimental implementation of Ramsey interferometry with photons. the Supplemental Material [40] for the spectral and temperature dependance of the background noise) and the pump wavelengths are set to $\lambda_{1}=1551.7 \mathrm{~nm}$ and $\lambda_{2}=1558.1 \mathrm{~nm}$ as a trade-off between good phase matching and weak spurious four-wave-mixing. The pulses are synchronized and overlapped with the signal photon in the nonlinear fiber using wavelength division multiplexer add-and-drop filters; the polarization of all three fields is aligned to be parallel. For a signal photon at $\lambda_{s}=1283.5 \mathrm{~nm}$, we first verified the power dependence of the conversion efficiency as shown in Fig. 3 using a decoy state source with an average of 0.1 photon/pulse. The conversion scales as a squared sine that mimics a Rabi oscillation. After correction for wavelengthdependent loss, the conversion efficiency reaches $90 \% \pm 5 \%$ limited by the power fluctuations of the pump beam and by higher-order leakage into other frequency modes due to the competing up-conversion process. This effect is most apparent at higher pump powers where there is a clear discrepancy between the expected coherent oscillation and the observed result.

To produce a tunable phase between the two states, we filtered the pump beams from the bichromatic photon and inserted a tunable free space delay on its path [Fig. 2(c)]. As indicated by Eq. (1), the BS-FWM process depends not only on the relative phase $\theta$ between the two spectral components of the single photon but also on the relative phase between the two pump fields. We must control those phase relationships independently since otherwise the precession acquired by the two pumps $[\theta$ in Eq. (1)] would exactly cancel out with the bichromatic qubit phase $\phi=2 \pi \Delta x\left(\nu_{s}-\nu_{i}\right) / c$ accumulated over the propagation distance $\Delta x$.

Figure 2(d) illustrates how the projective measurements are performed. First, the optical pump beams are filtered out using short pass filters, and the two spectral components of the bichromatic qubit are separated using commercially available wavelength division demultiplexers. The projective measurement is then made by performing single-photon detection on each arm using InGaAs avalanche photodiodes. Since our single-photon detectors can operate only in a gated mode, they are synchronized to match the arrival time of the single photons (typically using the heralding event). The photon flux and correlations at

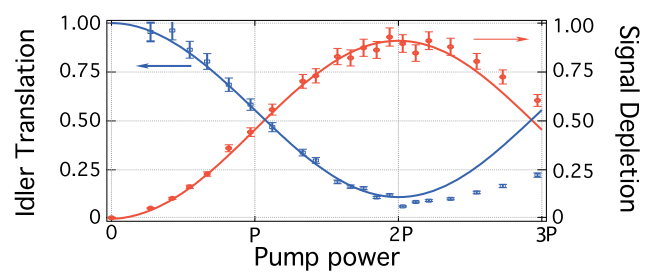

FIG. 3. Photon depletion and conversion efficiency to the idler frequency via BS FWM as a function of the pump power measured for decoy states with the setup depicted in Fig. 2(b). $P$ is the power corresponding to a polar $\pi / 2$ rotation on the Bloch sphere. 
the detectors are determined using a time-tagging module (coincidence acquisition).

Our approach allows generating any state of a bichromatic qubit and thus setting the bichromatic state of the photon anywhere on the Bloch sphere. The full setup consists of the four components discussed above (see Fig. 2) so that the photon is prepared in frequency state $\left|\nu_{s}\right\rangle$ (A), a $\pi / 2$ pulse is applied using the quantum frequency converter (B), a tunable phase is imparted on the bichromatic qubit (C), a $\pi / 2$ pulse is again applied using the same frequency converter (B) but in the reverse direction, and, lastly, the final state is reconstructed via frequency demultiplexing and single-photon detection (D). Experimentally, the BS-FWM pump power $P$ is adjusted to give a forward conversion efficiency of $1 / 2(P=2 \mathrm{~W})$ so that the bichromatic qubit exits the quantum frequency converter (B) as a balanced superposition of the two frequencies $\nu_{s}$ and $\nu_{i}$. In the tunable phase delay stage (C), the pump and signal arms are kept at nearly equal length so that the optical pulses temporally overlap with the reflected single photon when they are combined back in the nonlinear fiber. The resulting Ramsey interference is depicted in Fig. 4 showing the signal conversion to the idler frequency as a function of the imparted phase. As expected, the interference pattern exhibits fringes corresponding to the probabilities $p\left(\nu_{s}\right)=\sin (\phi / 2)$ and $p\left(\nu_{i}\right)=\cos (\phi / 2)$. The interference pattern shows fringes over a $\pi$ phase that corresponds to a free-space propagation of $0.36 \mathrm{~mm}$. The interference fringes are the proof that the underlying BS-FWM process preserves the coherence of the quantum fields. The visibility of the fringes is nearly $50 \%$ and is limited by the following two factors. The same nonlinear fiber and optical pulses are used for performing the first and second quantum frequency conversion but unfortunately the pump beams experience loss in the tunable delay element $(2 \mathrm{~dB})$ so that the second propagation results in a lesser conversion efficiency than the target of $\pi / 2$. Using a setup less sensitive to the loss experienced by the pump beams, the Ramsey interference visibility can reach 65\% (see Sec. S7 of the Supplemental Material for this alternative setup). Moreover, the bandwidth of the heralded single photon (unlike the decoy state used in Fig. 2) is nearly equal to the acceptance bandwidth of the BS FWM, which limits the maximum conversion to $80 \%$ (see Fig. S3 of the Supplemental Material for the

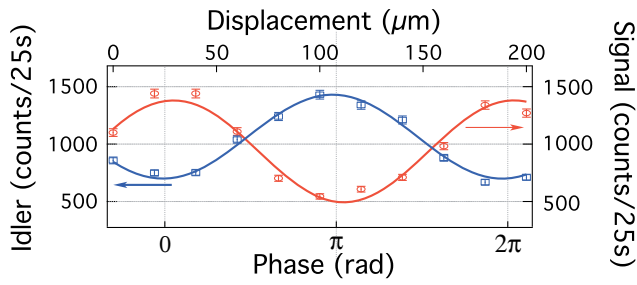

FIG. 4. Ramsey interference fringes observed while varying the phase between two BS-FWM frequency converters. conversion efficiency as a function of the pump power). For a detailed explanation of the impact of imperfect conversion efficiency on the fringe visibility, see Sec. S8 of the Supplemental Material [40]. We have also verified that our system is suitable for quantum information applications and preserves Fock states (i.e., technical noise does not spoil the fidelity), by measuring the second-order correlation function $g^{(2)}(t=0)$ of the output photon. This is achieved by replacing the demultiplexer by a balanced beam splitter in the projective measurement (D). We find that independently of whether we apply a transformation on the Bloch sphere or not (pump beams turned off), the $g^{(2)}(t=0)$ function remains at a value of 0.2 .

While the visibility of our interferogram does not reach unity, this demonstration proves that the quantum nature of the qubit is preserved. This visibility is sufficient to envisage applications even if they would require larger photon counting statistics than would an ideal unit visibility interferometer. Moreover, better visibility can be attained having more powerful laser pulses or dedicated nonlinear fibers. Indeed, this allows reducing the nonlinear interaction length and thus broadens the acceptance bandwidth of the FWM BS. Relaxing the phase matching condition would also allow a larger frequency difference between the two modes of the bichromatic photon. More powerful lasers would also allows us to use a nonattenuated copy of the pump pulse in our second frequency convertor rather than recycling the pump pulses from the first one.

We have a complete set of building blocks for encoding, manipulating, and measuring quantum information contained in frequency qubits. We have demonstrated that a single photon can be placed in a bichromatic state at any point on the corresponding Bloch sphere using a photonic Ramsey interferometer whose phase sensitivity is set by the beating wavelength $\Delta \lambda=c / \delta \nu$.

We believe our demonstration will find applications in quantum information. Indeed, bichromatic photons can constitute the interface between quantum systems operating at different frequencies [32-37,41] such as a quantum information carrier, quantum repeaters, or simply efficient detectors. For this purpose, much larger frequency difference could be achieved by adjusting the phase matching condition in a silica fiber as in our present realization or by translating our demonstration to a different system using sum or difference frequency generation that is better suited for such a wideband application. Recently, frequency translation between 980 and $1550 \mathrm{~nm}$ has been demonstrated using silicon nitride microcavities [42]. A bichromatic photon can also serve as a stable quantum information carrier. A potential extension of our work is the manipulation of entangled states rather than pure states. Photon pairs spontaneously generated on frequency combs [43] constitute an example of a high dimension frequency entangled state that could be manipulated using our Ramsey interferometer to perform quantum key distribution 
with improved robustness $[44,45]$. In addition, we foresee that single photon spectral-temporal pulse shaping [46] using a nearly identical setup as our Ramsey interferometer is another promising application as it may also serve as an interface between bandwidth-time unmatched quantum optics systems. Both these aspects are highly relevant to quantum key distribution [25,47-49] whose extension to longer distances will depend on quantum relays and overall robustness again loss and noise. Another class of applications may make use of the interferometer itself as our demonstration enables measuring a spectrally dependent phase change, i.e., performing quantitative phase spectroscopy $[50,51]$, with a very low amount of light. That is particularly relevant for performing spectroscopy on samples that are photosensitive or cannot tolerate any absorption such as eyes and phototrophs (organisms carrying out photosynthesis) and might even help studying the quantum nature of the photosynthesis process [52-54].

We acknowledge support from the Defense Advanced Research Projects Agency via the QuASAR program and the Air Force Office of Scientific Research under Grant No. FA9550-12-1-0377. S. R. is funded by a EU Marie Curie Fellowship (PIOF-GA-2012-329851).

*sclemmen@ulb.ac.be

[1] W. Laurence, New York Times, January 21, 1945, p. 34.

[2] A. D. Cronin, J. Schmiedmayer, and D. E. Pritchard, Rev. Mod. Phys. 81, 1051 (2009).

[3] L. Essen and J. Parry, Nature (London) 176, 280 (1955).

[4] N. F. Ramsey, Phys. Rev. 78, 695 (1950).

[5] A. D. Ludlow, M. M. Boyd, J. Ye, E. Peik, and P. O. Schmidt, Rev. Mod. Phys. 87, 637 (2015).

[6] C. J. Myatt, B. E. King, Q. A. Turchette, C. A. Sackett, D. Kielpinski, W. M. Itano, C. Monroe, and D. J. Wineland, Nature (London) 403, 269 (2000).

[7] M. Brune, E. Hagley, J. Dreyer, X. Maitre, A. Maali, C. Wunderlich, J. M. Raimond, and S. Haroche, Phys. Rev. Lett. 77, 4887 (1996).

[8] B. Pingault, J. N. Becker, C. H. H. Schulte, C. Arend, C. Hepp, T. Godde, A. I. Tartakovskii, M. Markham, C. Becher, and M. Atatüre, Phys. Rev. Lett. 113, 263601 (2014)

[9] G. Fuchs, V. Dobrovitski, D. Toyli, F. Heremans, and D. Awschalom, Science 326, 1520 (2009).

[10] C. G. Yale, B. B. Buckley, D. J. Christle, G. Burkard, F. J. Heremans, L. C. Bassett, and D. D. Awschalom, Proc. Natl. Acad. Sci. U.S.A. 110, 7595 (2013).

[11] F. Schmidt-Kaler, H. Häffner, M. Riebe, S. Gulde, G. P. Lancaster, T. Deuschle, C. Becher, C. F. Roos, J. Eschner, and R. Blatt, Nature (London) 422, 408 (2003).

[12] M. Kasevich and S. Chu, Phys. Rev. Lett. 67, 181 (1991).

[13] H. Müller, A. Peters, and S. Chu, Nature (London) 463, 926 (2010).

[14] H. Suchowski, G. Porat, and A. Arie, Laser Photonics Rev. 8, 333 (2014).

[15] J. Huang and P. Kumar, Phys. Rev. Lett. 68, 2153 (1992).
[16] Z. Ou, Phys. Rev. A 78, 023819 (2008).

[17] J.-M. Merolla, Y. Mazurenko, J.-P. Goedgebuer, and W. T. Rhodes, Phys. Rev. Lett. 82, 1656 (1999).

[18] L. Olislager, J. Cussey, A. T. Nguyen, P. Emplit, S. Massar, J.-M. Merolla, and K. Phan Huy, Phys. Rev. A 82, 013804 (2010).

[19] L. Olislager, E. Woodhead, K. Phan Huy, J.-M. Merolla, P. Emplit, and S. Massar, Phys. Rev. A 89, 052323 (2014).

[20] M. Bloch, S. W. McLaughlin, J.-M. Merolla, and F. Patois, Opt. Lett. 32, 301 (2007).

[21] S. Ramelow, L. Ratschbacher, A. Fedrizzi, N. K. Langford, and A. Zeilinger, Phys. Rev. Lett. 103, 253601 (2009).

[22] B. Brecht, D. V. Reddy, C. Silberhorn, and M. G. Raymer, Phys. Rev. X 5, 041017 (2015).

[23] D. V. Reddy, M. G. Raymer, and C. J. McKinstrie, Phys. Rev. A 91, 012323 (2015).

[24] D. V. Reddy, M. G. Raymer, and C. J. McKinstrie, Opt. Lett. 39, 2924 (2014).

[25] B. Brecht, A. Eckstein, R. Ricken, V. Quiring, H. Suche, L. Sansoni, and C. Silberhorn, Phys. Rev. A 90, 030302 (2014).

[26] T. Zhong, H. Zhou, R. D. Horansky, C. Lee, V. B. Verma, A. E. Lita, A. Restelli, J. C. Bienfang, R. P. Mirin, and T. Gerrits, New J. Phys. 17, 022002 (2015).

[27] P. Kumar, Opt. Lett. 15, 1476 (1990).

[28] H. J. McGuinness, M. G. Raymer, C. J. McKinstrie, and S. Radic, Phys. Rev. Lett. 105, 093604 (2010).

[29] A. S. Clark, S. Shahnia, M. J. Collins, C. Xiong, and B. J. Eggleton, Opt. Lett. 38, 947 (2013).

[30] K. Krupa, A. Tonello, V. V. Kozlov, V. Couderc, P. Di Bin, S. Wabnitz, A. Barthélémy, L. Labonté, and S. Tanzilli, Opt. Express 20, 27220 (2012).

[31] I. Agha, M. Davanço, B. Thurston, and K. Srinivasan, Opt. Lett. 37, 2997 (2012).

[32] A. P. VanDevender and P. G. Kwiat, J. Opt. Soc. Am. B 24, 295 (2007).

[33] R. V. Roussev, C. Langrock, J. R. Kurz, and M. Fejer, Opt. Lett. 29, 1518 (2004).

[34] P. Mataloni, O. Jedrkiewicz, and F. De Martini, Phys. Lett. A 243, 270 (1998).

[35] G. Giorgi, P. Mataloni, and F. De Martini, Phys. Rev. Lett. 90, 027902 (2003).

[36] M. A. Albota and F. N. Wong, Opt. Lett. 29, 1449 (2004).

[37] S. Tanzilli, W. Tittel, M. Halder, O. Alibart, P. Baldi, N. Gisin, and H. Zbinden, Nature (London) 437, 116 (2005).

[38] S. Zaske, A. Lenhard, C. A. Keßler, J. Kettler, C. Hepp, C. Arend, R. Albrecht, W.-M. Schulz, M. Jetter, P. Michler et al., Phys. Rev. Lett. 109, 147404 (2012).

[39] S. Ates, I. Agha, A. Gulinatti, I. Rech, M. T. Rakher, A. Badolato, and K. Srinivasan, Phys. Rev. Lett. 109, 147405 (2012).

[40] See Supplemental Material at http://link.aps.org/ supplemental/10.1103/PhysRevLett.117.223601 for details about noise mitigation, the characterization of our experiment and limitations of the setup.

[41] E. Diamanti, H. Takesue, T. Honjo, K. Inoue, and Y. Yamamoto, Phys. Rev. A 72, 052311 (2005).

[42] Q. Li, M. Davanço, and K. Srinivasan, Nat. Photonics 10, 406 (2016). 
[43] C. Reimer, L. Caspani, M. Clerici, M. Ferrera, M. Kues, M. Peccianti, A. Pasquazi, L. Razzari, B. E. Little, S. T. Chu et al., Opt. Express 22, 6535 (2014).

[44] L. Sheridan and V. Scarani, Phys. Rev. A 82, 030301 (2010).

[45] N. J. Cerf, M. Bourennane, A. Karlsson, and N. Gisin, Phys. Rev. Lett. 88, 127902 (2002).

[46] J. Sharping, Y. Okawachi, J. van Howe, C. Xu, Y. Wang, A. Willner, and A. Gaeta, Opt. Express 13, 7872 (2005).

[47] J. Mower, Z. Zhang, P. Desjardins, C. Lee, J. H. Shapiro, and D. Englund, Phys. Rev. A 87, 062322 (2013).

[48] I. Ali-Khan, C. J. Broadbent, and J. C. Howell, Phys. Rev. Lett. 98, 060503 (2007).
[49] S. P. Walborn, D. S. Lemelle, M. P. Almeida, and P. H. Souto Ribeiro, Phys. Rev. Lett. 96, 090501 (2006).

[50] N. Ji, V. Ostroverkhov, C.-Y. Chen, and Y.-R. Shen, J. Am. Chem. Soc. 129, 10056 (2007).

[51] M. Rinehart, Y. Zhu, and A. Wax, Biomed. Opt. Express 3, 958 (2012).

[52] S. F. Huelga and M. B. Plenio, Contemp. Phys. 54, 181 (2013).

[53] E. J. O'Reilly and A. Olaya-Castro, Nat. Commun. 5, 3012 (2014).

[54] E. Romero, R. Augulis, V. I. Novoderezhkin, M. Ferretti, J. Thieme, D. Zigmantas, and R. Van Grondelle, Nat. Phys. 10, 676 (2014). 